[18] G. Venema, Embeddings of compacta with shape dimension in the trivial range, Proc. Amer. Math. Soc. 55 (1976), 443-448.

19] C. T. C. Wall, Formal deformations, Proc. Lond. Math. Soc. (3) 16 (1966), 342-352

[20] J. H. C. Whitehead, Simplicial spaces, nuclei and m-groups, Proc. Lond. Math. Soc. (2) 45 (1939), 243-327.

FACHBEREICH MATHEMATIK

\section{UNIVERSITÄT-GRSAMTHOCHSCHULE STEGEN}

Hölderlinstr. 35900 Siegen

Federal Republic of Germany

\section{Mapping approximate inverse systems of compacta}

by

Sibe Mardešic (Zagreb) and Jack Segal (Seattle)

Abstract. Recently, T. Watahabe has extensively studied approximate mappings of inverse systems of spaces $f: X \rightarrow Y$. He showed :f $p: X \rightarrow X$ and $q: Y \rightarrow Y$ are ANR-resolutions of topologically complete spaces $X, Y$, then $f: X \rightarrow Y$ induces a mapping $f: X \rightarrow Y$ and conversely, every mapping $f: X \cdots \rightarrow Y$ is obtainaltic in this way. In this paper it is shown that the basic results of Watanabe's theory are valid also for approximate mappings of approximate inverse systems $\boldsymbol{X}, \boldsymbol{Y}$ of compact ANR's and compact Hausdorff spaces $X=\lim X, Y=\lim Y$. Approximate systems, newly introduced by S. Mardešć and L. R. Rubin, have bonding maps $p_{a a^{\prime}}, a \leqslant a^{\prime}$, where in general $p_{a_{1} a_{2} p_{a_{2} a_{3}}}$ differs from $p_{a_{1} a_{3}}$, but in a controlled way.

1. Introduction. An inverse system of spaces $X=\left(X_{a}, p_{a a^{\prime}}, A\right)$ (in the usual sense) consists of a directed set $(A, \leqslant)$, spaces $X_{a}, a \in A$, and maps $p_{a a^{\prime}}: X_{a^{\prime}} \rightarrow X_{a}, a \leqslant a^{\prime}$, such that $p_{a a}=\mathrm{id}$ and

$$
p_{a_{1} a_{2}} p_{a_{2} a_{3}}=p_{a_{1} a_{3}}, \quad a_{1} \leqslant a_{2} \leqslant a_{3} .
$$

The (usual) inverse limit $X=\lim X$ is the subspace $X \subseteq \Pi X_{a}$, which consists of all points $x=\left(x_{a}\right) \in I I X_{a}$ such that $p_{a a^{\prime}}\left(x_{a^{\prime}}\right)=x_{a}, a \leqslant a^{\prime}$. Projections $p_{a}: X \rightarrow X_{a}$ are restrictions to $X$ of the projections $\pi_{a}: \Pi X_{a} \rightarrow X_{a}, a \in A$.

A. mapping of systems $f: X \rightarrow Y=\left(Y_{b}, q_{b b^{\prime}}, B\right)$ consists of a function $f: B \rightarrow A$ and of mappings $f_{b}: X_{s(b)} \rightarrow Y_{b}, b \in B$, such that whenever $b_{1} \leqslant b_{2}$, there exists an index $a \in A, a \geqslant f\left(b_{1}\right), f\left(b_{2}\right)$, such that

$$
f_{b_{1}} p_{f\left(b_{1}\right) a}=q_{b_{1} b_{2}} f_{b_{2}} p_{s\left(b_{2}\right) a}
$$

(sce, e.g., [8], I, \$1.1). It is well known that for any mapping of systems $f=\left(f, f_{b}\right): X \rightarrow Y$ there is a unique mapping $f: X \rightarrow Y$ of the limits $X=\lim \boldsymbol{X}$, $Y=\lim Y$ such that

$$
f_{b} p_{S(b)}=q_{b} f^{\prime}, \quad b \in B
$$

This mapping is called the limit of $f$ and is denoted by $f=\lim f$ (see, e. g., $[8], I, \S 5.1$ ).

This paper was written during the Summer and Autumn Quarters of 1987, while S. Mardešic, on leave from the University of Zagreb, was visiting the University of Washington.

AMS(MOS) Subject Classification: 54B25, 54D 30, 54C55. 
If $f: X \rightarrow Y$ is a mapping between compact Hausdorff spaces, then there exist inverse systems $X, Y$ of compact ANR's (compact polyhedra) and there exists a map of systems $f: X \rightarrow Y$ such that $X=\lim X, Y=\lim Y$ and $f=\lim f$ (sec, e.g., [4] for the case when $X$ and $Y$ are metric and [3], [1] for the general case). However, if one chooses $\boldsymbol{Y}$ in advance, it may be impossible to find $X$ and $\boldsymbol{f}$ such that (3) holds for a given $f$. Such examples exist even when $X$ and $Y$ are metric compacta and $Y$ is a sequence of compact ANR's (polyhedra) (see, e.g., [2], [12] or [13]). This is the reason why various authors have studied more general mappings of systems $\boldsymbol{f}=\left(f, f_{b}\right): X \rightarrow Y$, where the commutativity relation (2) holds only approximately (see, e.g., [11] for the case of inverse sequences).

An extensive and very general study of this phenomenon is due to $T$. Watanabe (see [12], [13]). He combined the idea of approximate mapping with the idea of resolution, introduced in [3]. This enabled him to also consider non-compact spaces. However, all inverse systems in Watanabe's work are usual (commutative) systems, which satisfy (1).

Recently, S. Mardešić and L. R. Rubin have introduced and studied approximate inverse systems of metric compacta, i.e., systems where (1) holds only approximately [4]. These systems have proven very effective in constructing various compact Hausdorff spaces using polyhedra as terms of the system (see [4], [9] and [5]).

The purpose of the present paper is to establish all the basic features of Watanabe's theory also for approximate mappings between approximate systems of compact ANR's (polyhedra). In particular, such mappings $f: X \rightarrow Y$ induce a limit mapping $f: X \rightarrow Y$ between the limit spaces $X=\lim X, Y=\lim Y$ and conversely, every mapping $f: X \rightarrow Y$ between compact Hausdorff spaces is the limit of an approximate mapping $f: X \rightarrow \boldsymbol{Y}$ between two arbitrary approximate systems of compact ANR's (polyhedra) with $\lim X=X, \lim Y=Y$.

As a consequence of our general results, we can prove, e.g., the following. Every mapping $f: X \rightarrow Y$ between compact Hausdorff spaces with $\operatorname{dim} X \leqslant m$, $\operatorname{dim} Y \leqslant n$ is the limit of an approximate mapping $f: X \rightarrow Y$, where $\boldsymbol{X}$ and $\boldsymbol{Y}$ are approximate systems of polyhedra $X_{a}$ and $Y_{b}$ respectively with $\operatorname{dim} X_{a} \leqslant m$ and $\operatorname{dim} Y_{b} \leqslant n$. This is so because, by [4], every compact Hausdorff space $X$ with $\operatorname{dim} X \leqslant m$ is the limit of an approximate system of polyhedra of dimension $\leqslant m$.

If one insists on using commutative systems, such an expansion theorem in general does not hold, because there are compact Hausdorff spaces $Y$, dim $Y=1$, which are not representable as limits of 1-dimensional (commutative) inverse systems (see [4]).

In the last section of this paper we show that approximate systems of compact ANR's can be used in the shape theory of compact Hausdorff spaces,

We believe that the basic results of this paper can be generalized to topologically complete spaces, approximate systems of non-compact ANR's and (noncommutative) approximate resolutions.
2. Approximate inverse systems of compacta. We quote from [4] the basic definitions.

DEFINITXON 1. An approximate inverse system of metric compacta $X=\left(X_{a}, u_{a}, p_{a a^{\prime}}, A\right)$ consists of the following: An unbounded directed set $(A, \leqslant)$; for each $a \in A$, a compact metric space $X_{a}$ with metric $d_{a}=d$ and a real number $u_{a}>0$ (called a mesh); for each pair $a \leqslant a^{\prime}$ from $A$, a mapping $p_{a a^{\prime}}: X_{a^{\prime}} \rightarrow X_{a}$, satisfying the following conditions:

$$
\begin{aligned}
& d\left(p_{a_{1} a_{2}} p_{a_{2} a_{3}}, p_{a_{1} a_{3}}\right) \leqslant u_{a_{1}}, \quad a_{1} \leqslant a_{2} \leqslant a_{3} ; \quad p_{a a}=\mathrm{id} . \\
& \left(\forall a_{1} \in A\right)(\forall \eta>0)\left(\exists a_{1}^{\prime} \geqslant a_{1}\right)\left(\forall a_{3} \geqslant a_{2} \geqslant a_{1}^{\prime}\right) \\
& d\left(p_{a_{1} a_{2}} p_{a_{2} a_{3}}, p_{a_{1} a_{3}}\right) \leqslant \eta .
\end{aligned}
$$

(A3) $\quad(\forall a \in A)(\forall \eta>0)\left(\exists a^{\prime} \geqslant a\right)\left(\forall a^{\prime \prime} \geqslant a^{\prime}\right)\left(\forall x, x^{\prime} \in X_{a^{\prime \prime}}\right)$

$$
d\left(x, x^{\prime}\right) \leqslant u_{a^{\prime \prime}} \Rightarrow d\left(p_{a a^{\prime \prime}}(x), p_{a a^{\prime \prime}}\left(x^{\prime}\right)\right) \leqslant \eta .
$$

Here $d(f, g)=\sup d(f(x), g(x))$.

Defintrion 2. A point $x=\left(x_{a}\right) \in \Pi X_{a}$ belongs to $X=\lim \boldsymbol{X}$,

$$
X=\left(X_{a}, u_{a}, p_{a a^{\prime}}, A\right)
$$

provided, for every $a \in A$,

$$
x_{a}=\lim _{a^{\prime}} p_{a a^{\prime}}\left(x_{a^{\prime}}\right)
$$

The projections $p_{a}: X \rightarrow X_{a}$ are given by $p_{a}=\pi_{a} \mid X$.

We now quote (as propositions) several results from [4], [5], [9] and [10], which we need later. We assume that $X=\left(X_{a}, u_{a}, p_{a a^{\prime}}, A\right)$ is an approximate system of metric compacta with limit $X$ and projections $p_{a}: X \rightarrow X_{a}, a \in A$.

Propositron 1. If $X$ is a commutative system, then $X=\lim X$ as defined in Definition 2, coincides with the usual inverse limit (see [4], Proposition 1).

Propostrion 2. If $X=\left(X_{a}, p_{a a^{\prime}}, A\right)$ is a commutative system of metric compacta and $A$ is unbounded (has no maximal element) and is cofinite (every element has only finitely many predecessors), then there exist numbers $u_{a}>0, a \in A$, such that $\left(X_{a}, u_{a}, p_{a a}, A\right)$ is an approximate system ([4], Remark 2).

Proposinion 3. If $X_{a} \neq \varnothing$ for all $a \in A$, then $X=\lim X$ is a compact Hausdorff space and $X \neq \varnothing$ (sce [4], Theorem 1 and 2).

Proposition 4. For every $a \in A$,

(see [4], Lemma 4).

$$
\lim _{a^{\prime}} d\left(p_{a a^{\prime}} p_{a^{\prime}}, p_{a}\right)=0
$$


Proposimon 5. Let $X=\left(X_{a}, u_{a}, p_{a a^{\prime}}, A\right)$ be an approximate system with limit $X$ and projections $p_{a}$. Let $<^{\prime}$ be a binary relation on $A$ such that

$$
a_{1}<a_{2} \Rightarrow a_{1}<a_{2},
$$

$a_{1}<{ }^{\prime} a_{2}$ and $a_{2} \leqslant a_{3} \Rightarrow a_{1}<^{\prime} a_{3}$,

$(\forall a \in A)\left(\exists a^{\prime} \in A\right) a<{ }^{\prime} a^{\prime}$.

Moreover, let $a_{1} \leqslant^{\prime} a_{2}$ mean that $a_{1}<^{\prime} a_{2}$ or $a_{1}=a_{2}$. Then $A^{\prime}=\left(A, \leqslant^{\prime}\right)$ is $a$ directed set and $X^{\prime}=\left(X_{a}, u_{a}, p_{a a^{\prime}}, A^{\prime}\right)$ is an approximate system with limit $X^{\prime}=X$ and projections $p_{a}^{\prime}=p_{a}$ (see [5]; Proposition 9).

Propositron 6. The following statements hold:

(B1) Let $a \in A$ and let $U \subseteq X_{a}$ be an open set which contains $p_{a}(X)$. Then there is an $a^{\prime} \geqslant a$ such that $p_{a a^{\prime \prime}}\left(X_{a^{\prime \prime}}\right) \subseteq U$ for each $a^{\prime \prime} \geqslant a^{\prime}$.

(B2) For every open covering $\%$ of $X$ there exists an $a \in A$ such that for any $a_{1} \geqslant 0$ there exists an open covering $\mathscr{V}$ of $X_{a_{1}}$ for which $\left(p_{a_{1}}\right)^{-1}(\mathscr{V})$ refines $\mathscr{U}$ (see [4], Theorem 3 and [9], Theorem 1).

PROPOSITION 7. The following statements hold:

(R1) For every compact ANR $P, \eta>0$ and mapping $h: X \rightarrow P$, there is an $a \in A$ such that for any $a^{\prime} \geqslant a$ there is a mapping $f: X_{a^{\prime}} \rightarrow P$ for which $d\left(f p_{a^{\prime}}, h\right) \leqslant 2 \eta$.

(R2) Let $P$ be a compact ANR and $\eta>0$. Whenever $a \in A$ and $f, f^{\prime}: X_{a} \rightarrow P$ are mappings with the property $d\left(f p_{a}, f^{\prime} p_{a}\right)<\eta$, then there is an $a^{\prime} \in A$ such that for any $a^{\prime \prime} \geqslant a^{\prime}$ one has $d\left(f p_{a a^{\prime \prime}}, f^{\prime} p_{a a^{\prime \prime}}\right)<\eta$.

(R1) was proven in [5] as Proposition 7. (R2) is an immediate consequence of (B2).

Definition 3 [10]. An almost commutative system $X=\left(X_{a}, p_{a \prime}, A\right)$ consists of an unbounded directed set $(A, \leqslant)$, of metric compacta $X_{a}$ and of maps $p_{a a^{\prime}}$ such that it is possible to associate with every $a \in A$ a number $u_{a}>0$ so as to obtain an approximate system as in Definition 1 . Such numbers $u_{a}$ are called admissible meshes.

Remark 1. If $\left(u_{a}\right)$ and $\left(u_{a}^{\prime}\right)$ are admissible meshes for the same almost commutative system and $X=\left(X_{a}, u_{a}, p_{a a^{\prime}}, A\right), \quad X^{\prime}=\left(X_{a}, u_{a}^{\prime}, p_{a a^{\prime}}, A\right)$, then $X=\lim X$ coincides with $X^{\prime}=\lim X^{\prime}$, because the limit space does not depend on the meshes (see Definition 2). Therefore, the limit space of an almost commutative system is well defined.

Remark 2. If $u_{a}$ and $u_{a}^{\prime}$ are admissible meshes for the same almost commutative system, then $\max \left(u_{a}, u_{a}^{\prime}\right)$ and $\min \left(u_{a}, u_{a}^{\prime}\right)$ are also admissible meshes for that system.

Proposition 8. For every approximate system $X=\left(X_{a}, u_{a}, p_{a a^{\prime}}, A\right)$ over $a$ cofinite set $A$ there exist admissible meshes $u_{a}^{\prime}>u_{a}, a \in A$ (see [10], Lemma 1).

\section{Mappings of approximate systems and their limits.}

Definition 4. Let $X=\left(X_{a}, u_{a}, p_{a a^{\prime}}, A\right)$ and $Y=\left(Y_{b}, v_{b}, q_{b b^{\prime}}, B\right)$ be approximate systems of metric compacta. A mapping of approximate systems $f: X \rightarrow Y$ (or approximate mapping) consists of a function $f: B \rightarrow A$ and of mappings $f_{b}: X_{f^{(}(b)} \rightarrow Y_{b}$. $b \in B$, such that whenever $b \leqslant b^{\prime}$, then there exists an index $a \in A, a \geqslant f(b), f\left(b^{\prime}\right)$, such that for every $a^{\prime} \geqslant a$ the following condition holds

$$
d\left(f_{b} p_{f(b) a^{\prime}}, q_{b b^{\prime}} f_{b^{\prime}} p_{f\left(b^{\prime}\right) a^{\prime}}\right) \leqslant v_{b} .
$$

(see [12], $\S 2$, condition (AM2))

The main purpose of this section is to prove the following theorem.

THeOREM 1. If $\boldsymbol{f}=\left(f, f_{b}\right): X \rightarrow Y$ is a mapping of approximate systems, then there exists a unique mapping $f: X \rightarrow Y, X=\lim X, Y=\lim Y$, such that

$$
d\left(f_{b} p_{f(b)}, q_{b} f\right) \leqslant v_{b}, \quad b \in B .
$$

The mapping $f: X \rightarrow Y$ is called the limit of $f$ and is denoted by $f=\lim f$. (It should not be confused with $f: B \rightarrow A$ from $f=\left(f, f_{b}\right): X \rightarrow Y$.)

To prove the existence of $f$ we need the following lemma.

LeMMA 1. Let $X$ be a compact Hausdorff space and let $h_{b}: X \rightarrow Y_{b}, b \in B$, be maps such that

$$
d\left(h_{b}, q_{b b^{\prime}} h_{b^{\prime}}\right) \leqslant v_{b}, \quad b \leqslant b^{\prime} .
$$

Then there exists a mapping $f: X \rightarrow Y=\lim Y$ such that

$$
d\left(h_{b}, q_{b} f\right) \leqslant v_{b}, \quad b \in B .
$$

Proof of Lemma 1. For a given $b \in B$ we consider all $b^{\prime} \geqslant b$ and the maps $q_{b b^{\prime}} h_{b}: X \rightarrow Y_{b}$. We will show that these maps form a Cauchy net and therefore

is a well-defined map $f^{b}: X \rightarrow Y_{b}$.

Indeed, for any $\eta>0$, by (A2), there exists an index $b^{\prime} \geqslant b$ such that

$$
d\left(q_{b b_{1},} q_{b_{1} b_{3}}, q_{b b_{3}}\right) \leqslant \eta, \quad b^{\prime} \leqslant b_{1} \leqslant b_{3} .
$$

Moreover, by (A3), one can assume that, for any $b^{\prime \prime} \geqslant b^{\prime}$,

$$
d\left(y, y^{\prime}\right) \leqslant v_{b^{\prime \prime}} \Rightarrow d\left(q_{b b^{\prime \prime}}(y), q_{b b^{\prime \prime}}\left(y^{\prime}\right)\right) \leqslant \eta \text {. }
$$

(1) and $_{4}(5)$ imply

$$
d\left(q_{b b_{1}} h_{b_{1}}, q_{b b_{1}} q_{b_{1} b_{3}} h_{b_{3}}\right) \leqslant \eta, \quad b^{\prime} \leqslant b_{1} \leqslant b_{3},
$$

which together with (4) yields

$$
d\left(q_{b b_{1}} h_{b_{1},}, q_{b b_{3}} h_{b_{3}}\right) \leqslant 2 \eta, \quad b^{\prime} \leqslant b_{1} \leqslant b_{3}:
$$

Analogously, we obtain

Since $B$ is directed, (7) and $(8)$ prove

$$
d\left(q_{b b_{1}} h_{b_{1}}, q_{b b_{2}} h_{b_{2}}\right) \leqslant 4 \eta, \quad b^{\prime} \leqslant b_{1}, b_{2},
$$


which proves that $\left(q_{b b^{\prime}}, h_{b^{\prime}}, b^{\prime} \geqslant b\right)$ is, indeed, a Cauchy net of mappings $X \rightarrow Y_{b}$. For every $x \in X$ and $b^{\prime} \leqslant b_{1} \leqslant b_{3}$, by (4), we have

$$
d\left(q_{b b_{1}} q_{b_{1} b_{3}} h_{b_{3}}(x), q_{b b_{3}} h_{b_{3}}(x)\right) \leqslant \eta .
$$

Passing to the limit with $b_{3}$, we obtain

$$
d\left(q_{b b_{1}} f^{b_{1}}(x), f^{b}(x)\right) \leqslant \eta, \quad b_{1} \geqslant b^{\prime}
$$

which shows that

$$
f^{b}(x)=\lim _{b_{1}} q_{b b_{x}} f^{b_{1}}(x) .
$$

This means that the mappings $f^{b}: X \rightarrow Y_{b}, b \in B$, determine a mapping $f: X \rightarrow Y$ $\subseteq \Pi Y_{b}$ such that

$$
q_{b} f=f^{b}, \quad b \in B .
$$

Passing to the limit with $b^{\prime}$ in (1), we obtain

$$
d\left(h_{b}, f^{b}\right) \leqslant v_{b}, \quad b \in B,
$$

which, by (13), coincides with the desired formula (2).

Proof of Theorem 1. Existence. Given a mapping of approximate systems $f=\left(f, f_{b}\right): X \rightarrow Y$, we put

$$
h_{b}=f_{b} p_{f(b)}, \quad b \in B .
$$

By Lemma 1, it suffices to show that the maps $h_{b}: X \rightarrow Y_{b}, b \in B$, satisfy (1)

By Definition 4, if $b \leqslant b^{\prime}$, there is an $a \geqslant f(b), f\left(b^{\prime}\right)$ such that (AM1) holds for all $a^{\prime} \geqslant a$. This implies

$$
d\left(f_{b} p_{f(b) a^{\prime}} p_{a^{\prime}}, q_{b^{\prime}} f_{b^{\prime}}, p_{f\left(b^{\prime}\right) a^{\prime}} p_{a^{\prime}}\right) \leqslant v_{b} .
$$

Passing to the limit with $a^{\prime}$, we obtain (1), because, by Proposition 4, for any $a \in A$, one has

$$
\lim _{a^{\prime}} p_{a a^{\prime}} p_{a^{\prime}}=p_{a} .
$$

The uniqueness of $f$ is a consequence of the following lemma.

LRMMA 2. Let $X$ be a compact Hausdorff space and let $h_{b}, h_{b}^{\prime}: X \rightarrow Y_{b}, b \in B$, be two families of mappings satisfying

$$
d\left(h_{b}, h_{b}^{\prime}\right) \leqslant v_{b}, \quad b \in B .
$$

Let $f, f^{\prime}: X \rightarrow X$ be mappings such that $f$ satisfies (2) and $f^{\prime}$ satisfies the analogous relations with $h_{b}^{\prime}$. Then $f=f^{\prime}$.
Proof of Lemma 2. Given any $b \in B$ and $\eta>0$, by Proposition 4, there is an index $b^{\prime} \geqslant b$ such that

$$
d\left(q_{b b^{\prime \prime}} q_{b^{\prime \prime}}, q_{b}\right) \leqslant \eta, \quad b^{\prime \prime} \geqslant b^{\prime} .
$$

By (A3), one can assume that for $b^{\prime \prime} \geqslant b^{\prime}(5)$ also holds. Then, by (2), one has

$$
d\left(q_{b b^{\prime \prime}} h_{b^{\prime \prime}}, q_{b b^{\prime \prime}} q_{b^{\prime \prime}} f\right) \leqslant v_{b}, \quad b^{\prime \prime} \geqslant b^{\prime} .
$$

Analogously,

$$
d\left(q_{b b^{\prime \prime}} h_{b^{\prime \prime}}^{\prime}, q_{b b^{\prime \prime}} q_{b^{\prime \prime}} f^{\prime}\right) \leqslant \eta_{b}, \quad b^{\prime \prime} \geqslant b^{\prime}
$$

Furthermore, (5) and (18) imply

$$
d\left(q_{b b^{\prime \prime}} h_{b^{\prime \prime}}, q_{b b^{\prime \prime}} h_{b^{\prime \prime}}^{\prime}\right) \leqslant \eta, \quad b^{\prime \prime} \geqslant b^{\prime} .
$$

Now, (19), (20), (22), (21) and again (19) yield

$$
d\left(q_{b} f, q_{b} f^{\prime}\right) \leqslant 5 \eta, \quad b \in B .
$$

Since $\eta>0$ was arbitrary, we conclude that $q_{b} f=q_{b} f^{\prime}$, for all $b \in B$, and, therefore, $f=f^{\prime}$.

Proof of Theorem 1. Uniqueness. It suffices to apply Lemma 2 to $h_{b}=h_{b}^{\prime}$ $=f_{b} p_{S(b)}$ and $f, f^{\prime}$.

Definition 5. An almost commutative mapping $f: X \rightarrow Y$ between almost commutative systems consists of a function $f: B \rightarrow A$ and of nappings $f_{b}: X_{f(b)} \rightarrow Y_{b}$, $b \in B$, such that $f=\left(f, f_{b}\right)$ is an approximatc mapping for some choice of admissible meshes. The limit mapping $f: X \rightarrow Y$ is defined as the limit of such an approximate mapping.

Remark 3. Let $v_{b}$ and $v_{b}^{\prime}, b \in B$, be admissible meshes of an almost commutative system $\left(Y_{b}, q_{b b^{\prime}}, B\right)$ and let $f=\left(f, f_{b}\right)$ be an approximate mapping into both $\boldsymbol{Y}=\left(Y_{b}, v_{b}, q_{b b^{\prime}}, B\right)$ and $\boldsymbol{Y}^{\prime}=\left(Y_{b}, v_{b}^{\prime}, q_{b b^{\prime}}, B\right)$ with limits $f$ and $f^{\prime}$ respectively. Clearly, $f$ is also an approximate mapping into $\left(Y_{b}, \max \left(v_{b}, v_{b}^{\prime}\right), q_{b b^{\prime}}, B\right)$. Moreover,

$$
\begin{aligned}
& d\left(f_{b} p_{S(b)}, q_{b} f\right) \leqslant v_{b} \leqslant \max \left(v_{b}, v_{b}^{\prime}\right), \\
& d\left(f_{b} p_{S(b)}, q_{b} f^{\prime}\right) \leqslant v_{b}^{\prime} \leqslant \max \left(v_{b}, v_{b}^{\prime}\right) .
\end{aligned}
$$

By the uniqueness in Theorem 1 , one concludes that $f=f^{\prime}$, so that $\lim f \operatorname{does}$ not depend on the choice of admissible meshes.

Remark 4. In some cases an approximate mapping of systems $f=\left(f, f_{b}\right): X \rightarrow Y$ also satisfies the additional condition

(AM2) $\quad(\forall b \in B)(\forall \eta>0)\left(\exists b^{\prime} \geqslant b\right)\left(\forall b^{\prime \prime} \geqslant b^{\prime}\right)\left(\exists a \geqslant f(b), f\left(b^{\prime \prime}\right)\right)\left(\forall a^{\prime} \geqslant a\right)$ $d\left(f_{b} p_{f(b) a^{\prime}}, q_{b b^{\prime \prime}} f_{b^{\prime \prime}} p_{f\left(b^{\prime \prime}\right) a^{\prime}}\right) \leqslant \eta$. 
In this case the limit map $f: X \rightarrow Y$ satisfies the commutative relation

$$
f_{b} p_{f(b)}=q_{b} f, \quad b \in B .
$$

Indeed, by (13), (3) and 15 ,

$$
q_{b} f=\lim _{b^{\prime \prime}} q_{b b^{\prime \prime}} f_{b^{\prime \prime}} p_{f\left(b^{\prime \prime}\right)} .
$$

Therefore, Proposition 4 and (AM2) imply.

$$
d\left(f_{b} p_{s(b)}, q_{b} f\right) \leqslant \eta, \quad b \in B .
$$

Since $\eta>0$ was arbitrary, (28) proves (26).

4. Representing mappings as limits of approximate mappings. In this section we prove the following expansion theorem.

THEOREM 2. Let $X$ and $Y$ be almost commutative systems with limits $X$ and $X$ respectively and let $f: X \rightarrow Y$ be a mapping. If all $Y_{b}, b \in B$, are compact ANR's and $B$ is cofinite, then there exists an almost commutative mapping $f: X \rightarrow Y$ such that $f=\lim f$.

We first prove a lemma which often enables us to verify that a collection of maps is an approximate mapping.

Lemma 3. Let $\boldsymbol{X}$ and $\boldsymbol{Y}=\left(Y_{b}, v_{b}, q_{b b^{\prime}}, B\right)$ be approximate systems, let $f: B \rightarrow A$ be a function and let $f_{b}: X_{f(b)} \rightarrow Y_{b}, b \in B$, be maps which satisfy the following condition:

$$
d\left(f_{b} p_{f(b)}, q_{b b^{\prime}} f_{b^{\prime}} p_{f\left(b^{\prime}\right)}\right) \leqslant v_{b}, \quad b \leqslant b^{\prime} .
$$

Then $f=\left(f, f_{b}\right)$ is a mapping of approximate systems $f_{j} X \rightarrow Y^{\prime}=\left(Y_{b}, v_{b}^{\prime}, q_{b b^{\prime}}, B\right)$, for any' choice of admissible meshes $v_{b}^{\prime}>v_{b}, b \in B$.

Proof. Given $b_{1} \leqslant b_{2}$ we must find an index $a^{\prime} \in A, a^{\prime} \geqslant f\left(b_{1}\right), f\left(b_{2}\right)$, such that for all $a^{\prime \prime} \geqslant a^{\prime}$ one has

$$
\text { (2) } \quad d\left(f_{b_{1}} p_{f\left(b_{1}\right) a^{\prime \prime}}, q_{b_{1} b_{2}} f_{b_{2}} p_{f\left(b_{2}\right) a^{\prime \prime}}\right) \leqslant v_{b_{1}}^{\prime} \text {. }
$$

Choose numbers $\eta_{1}>0, \eta_{2}>0$ such that

$$
\begin{gathered}
\text { (3) } \quad d\left(x, x^{\prime}\right) \leqslant \eta_{1} \Rightarrow d\left(f_{b_{1}}(x), f_{b_{1}}\left(x^{\prime}\right)\right) \leqslant \frac{1}{5}\left(v_{b_{1}}^{\prime}-v_{b_{1}}\right) . \\
\text { (4) } \quad d\left(x, x^{\prime}\right) \leqslant \eta_{2} \Rightarrow d\left(q_{b_{1} b_{2}} f_{b_{2}}(x), q_{b_{1} b_{2}} f_{b_{2}}\left(x^{\prime}\right)\right) \leqslant \frac{1}{5}\left(v_{b_{1}}^{\prime}-v_{b_{1}}\right) .
\end{gathered}
$$

By (A2), there is an index $a \geqslant f\left(b_{1}\right), f\left(b_{2}\right)$ such that for any $a^{\prime \prime} \geqslant a$ one has

$$
d d\left(p_{f\left(b_{l}\right) a} p_{a a^{\prime \prime}}, p_{\left.f\left(b_{l}\right) a^{\prime \prime}\right)} \leqslant \eta_{l}, \quad i=1,2 .\right.
$$

Now (3) and (4) imply

$$
\begin{array}{cc}
(6) & d\left(f_{b_{1}} p_{f\left(b_{1}\right) a} p_{a a^{\prime \prime}}, f_{b_{1}} p_{f\left(b_{1}\right) a^{\prime \prime}}\right) \leqslant \frac{1}{3}\left(v_{b_{1}}^{\prime}-v_{b_{1}}\right), \\
(7) & d\left(q_{b_{1} b_{2}} f_{b_{2}} p_{f\left(b_{2}\right) a} p_{a a^{\prime \prime}}, q_{b_{1} b_{2}} f_{b_{2}} p_{S\left(b_{b_{3}}\right) a^{\prime \prime}}\right) \leqslant \frac{1}{5}\left(v_{b_{1}}^{\prime}-v_{b_{1}}\right) ;
\end{array}
$$

Since $\lim _{a^{\prime \prime}} p_{a a^{\prime \prime}, p_{a^{\prime \prime}}}=p_{a}$ and $\lim _{a^{\prime \prime}} p_{f\left(b_{i}\right) a^{\prime \prime}} p_{a^{\prime \prime}}=p_{f\left(b_{i}\right)}, i=1,2$ (Proposition 4), (6) and (7) yield

$$
\begin{gathered}
d\left(f_{b_{1}} p_{f\left(b_{1}\right) a} p_{a}, f_{b_{1}} p_{f\left(b_{1}\right)}\right) \leqslant \frac{1}{5}\left(v_{b_{1}}^{\prime}-v_{b_{1}}\right), \\
d\left(q_{b_{1} b_{2}} f_{b_{2}} p_{f\left(b_{2}\right) a} p_{a}, q_{b_{1} b_{2}} f_{b_{2}} p_{f\left(b_{2}\right)}\right) \leqslant \frac{1}{5}\left(v_{b_{1}}^{\prime}-v_{b_{1}}\right) .
\end{gathered}
$$

Applying the assumption (1), we conclude that

$$
d\left(f_{b_{1}} p_{S\left(b_{1}\right) a} p_{a}, q_{b_{1} b_{2}} f_{b_{2}} p_{S\left(b_{2}\right) a} p_{a}\right) \leqslant \frac{2}{5}\left(v_{b_{1}}^{\prime}-v_{b_{1}}\right)+v_{b_{1}} .
$$

By continuity, there is a neighborhood $U$ of $p_{a}(X)$ in $X_{a}$ such that

$$
d\left(f_{b_{1}} p_{f\left(b_{1}\right) a}\left|U, q_{b_{1} b_{2}} f_{b_{2}} p_{f\left(b_{2}\right) a}\right| U\right) \leqslant \frac{3}{5}\left(v_{b_{1}}^{\prime}-v_{b_{1}}\right)+v_{b_{1}} .
$$

We now use property (B1) (Proposition 6) to conclude that there is an $a^{\prime} \geqslant a$ such that for any $a^{\prime \prime} \geqslant a^{\prime}$ one has

Therefore, (11) ;yields

$$
p_{a a^{\prime \prime}}\left(X_{a^{\prime \prime}}\right) \subseteq U
$$

(13) $\quad d\left(f_{b_{1}} p_{S\left(b_{1}\right) a} p_{a a^{\prime \prime}}, q_{b_{1} b_{2}} f_{b_{2}} p_{f\left(b_{2}\right) a} p_{a a^{\prime \prime}}\right) \leqslant \frac{3}{3}\left(v_{b_{1}}^{\prime}-v_{b_{1}}\right)+v_{b_{1}}, \quad a^{\prime \prime} \geqslant a^{\prime}$.

By (6), (7) and (13), we conclude that (2) indeed holds for all $a^{\prime \prime} \geqslant a^{\prime}$.

Proof of Theorem 2. By assumption there are numbers $v_{b}>0, b \in B$, such that $Y=\left(Y_{b}, v_{b}, q_{b b^{\prime}}, B\right)$ is an approximate system. By Proposition 8, there exist admissible meshes $v_{b}^{\prime}>v_{b}, b \in B$. By Lemma 3 , it suffices to produce a function $f: B \rightarrow A$ and mappings $f_{b}: X_{f(b)} \rightarrow Y_{b}, b \in B$, such that

$$
\begin{gathered}
d\left(f_{b} p_{f(b)}, q_{b b^{\prime}} f_{b^{\prime}} p_{f\left(b^{\prime}\right)}\right) \leqslant v_{b}^{\prime}, \\
d\left(f_{b} p_{f(b)}, q_{b} f\right) \leqslant v_{b}^{\prime}, \quad b \in B .
\end{gathered}
$$

First note that (A1) implies

$$
d\left(q_{b b^{\prime}} q_{b^{\prime} b^{\prime \prime}} q_{b^{\prime \prime}}, q_{b b^{\prime \prime}} q_{b^{\prime \prime}}\right) \leqslant v_{b}, \quad b \leqslant b^{\prime} \leqslant b^{\prime \prime} .
$$

Passing to the limit with $b^{\prime \prime}$, we obtain (by Proposition 4)

$$
d\left(q_{b b^{\prime}} q_{b^{\prime}}, q_{b}\right) \leqslant v_{b}, \quad b \leqslant b^{\prime} .
$$

By uniform continuity and cofiniteness of $B$, for any $b \in B$ there is an $\eta_{b}>0$ such that for all $b_{0} \leqslant b$ one has

$$
d\left(y, y^{\prime}\right) \leqslant \eta_{b} \Rightarrow d\left(\boldsymbol{q}_{b_{0} b}(y), q_{p_{0} b}\left(y^{\prime}\right)\right) \leqslant \frac{1}{2}\left(v_{b_{0}}^{\prime}-v_{b_{0}}\right) .
$$

Since $Y_{b}, b \in B$, is an ANR, property (R, (see Proposition 7) yields an $a=f(b) \in A$ and a mapping $f_{b}: X_{f(b)} \rightarrow Y_{b}^{\prime}$ such that

$$
d\left(f_{b} p_{S(b)}, q_{b} f\right) \leqslant \min \left\{\eta_{b}, \frac{1}{2}\left(v_{b}^{\prime}-v_{b}\right), v_{b}^{\prime}\right\} .
$$


If $b \leqslant b^{\prime}$, then (19) and (18) yield

$$
d\left(q_{b b^{\prime}}, f_{b^{\prime}} p_{f\left(b^{\prime}\right)}, q_{b b^{\prime}}, q_{b^{\prime}} f\right) \leqslant \frac{1}{2}\left(v_{b}^{\prime}-v_{b}\right) .
$$

Now, (19), (17) and (20) yield (14). Moreover (19) implies (15).

5. Contiguous and equivalent approximate mappings. Different approximate mappings can have the same limit mapping. In this section we study this phenomenon.

Definition 6. Let $X$ and $\boldsymbol{Y}=\left(Y_{b}, v_{b}, q_{b b^{\prime}}, B\right)$ be approximate systems. Two approximate mappings $f=\left(f, f_{b}\right), f^{\prime}=\left(f^{\prime}, f_{b}^{\prime}\right): X \rightarrow Y$ are called contiguous, denoted by $f \equiv f^{\prime}$, provided for every $b \in B$ there is an $a \in A, a \geqslant f(b), f^{\prime}(b)$, such that for any $a^{\prime} \geqslant a$ one has

$$
d\left(f_{b} p_{f(b) a^{\prime}}, f_{b}^{\prime} p_{f^{\prime}(b) a^{\prime}}\right) \leqslant v_{b}
$$

Two almost commutative maps $f, f^{\prime}: X \rightarrow Y$ are called contiguous provided they are contiguous as approximate mappings for some choice of admissible meshes $v_{b}>0, b \in B$.

The following is an easy consequence of Lemma 2 .

THBorem 3. Let $\boldsymbol{f}, \boldsymbol{f}^{\prime}: \boldsymbol{X} \rightarrow \boldsymbol{Y}$ be approximate maps of systems with limits $f, f^{\prime}: X \rightarrow Y$ respectively. If $f$ and $f^{\prime}$ are contiguous, then $f=f^{\prime}$.

Proof. Let $h_{b}=f_{b} p_{f(b)}, h_{b}^{\prime}=f_{b}^{\prime} p_{f^{\prime}(b)}$. By (LM), $f$ satisfies $\S 3(2)$ and $f^{\prime}$ satisfies the analogous formula. By Lemma 2 , it suffices to show that $\S 3(18)$ also holds.

By (1), we have

$$
d\left(f_{b} p_{S(b) a^{\prime}} p_{a^{\prime}}, f_{b}^{\prime} p_{S^{\prime}(b) a^{\prime}} p_{a^{\prime}}\right) \leqslant v_{b},
$$

for $a^{\prime}$ sufficiently large. Passing to the limit with $a^{\prime}$, by $\$ 3(17)$, we indeed obtain $\S 3(18)$.

The following lemma is sometimes used to conclude that two approximate mappings are contiguous.

LemMa 4. Let $X$ and $Y=\left(Y_{b}, v_{b}, q_{b b^{\prime}}, B\right)$ be approximate systems and let $f=\left(f, f_{b}\right), f^{\prime}=\left(f^{\prime}, f_{b}^{\prime}\right): X \rightarrow Y$ be approximate maps. If

$$
d\left(f_{b} p_{f(b)}, f_{b}^{\prime} p_{f^{\prime}(b)}\right) \leqslant v_{b}, \quad b \in B,
$$

then $f \equiv f^{\prime}: X \rightarrow Y^{\prime}=\left(Y_{b}, v_{b}^{\prime}, q_{b b^{\prime}}, B\right)$, for any choice of admissible meshes $v_{b}^{\prime}>v_{b}$, $b \in B$.

Proof. The proof is similar to the proof of Lemma 3. By (A2), there is an index $a \geqslant f(b), f^{\prime}(b)$ such that for any $a^{\prime \prime} \geqslant a$ one has

$$
\begin{aligned}
& d\left(f_{b} p_{S(b) a} p_{a a^{\prime \prime}}, f_{b} p_{S(b) a^{\prime \prime}}\right) \leqslant \frac{1}{5}\left(v_{b}^{\prime}-v_{b}\right), \\
& d\left(f_{b}^{\prime} p_{f^{\prime}(b) a} p_{a a^{\prime \prime}}, f_{b}^{\prime} p_{f^{\prime}(b) a^{\prime \prime}}\right) \leqslant \frac{1}{5}\left(v_{b}^{\prime}-v_{b}\right) .
\end{aligned}
$$

Since $\lim _{\alpha^{\prime \prime}} p_{a a^{\prime \prime}} p_{a^{\prime \prime}}=p_{a}($ Proposition 3), (4) and (5) imply

$$
\begin{aligned}
& d\left(f_{b} p_{f(b) a} p_{a}, f_{b} p_{f(b)}\right) \leqslant \frac{1}{5}\left(v_{b}^{\prime}-v_{b}\right), \\
& d\left(f_{b}^{\prime} p_{f^{\prime}(b) a} p_{a}, f_{b}^{\prime} p_{f^{\prime}(b)}\right) \leqslant \frac{1}{5}\left(v_{b}^{\prime}-v_{b}\right) .
\end{aligned}
$$

Using (3), one obtains

$$
d\left(f_{b} p_{f^{\prime}(b) a p_{a}}, f_{b}^{\prime} p_{f^{\prime}(b) a} p_{a}\right) \leqslant \xi_{s^{\prime}}^{2}\left(v_{b}^{\prime}-v_{b}\right)+v_{b} .
$$

By continuity, there is a neighborhood $U$ of $p_{a}(X)$ in $X_{a}$ such that

$$
d\left(f_{b} p_{f(b) a}\left|U, f_{b}^{\prime} p_{f^{\prime}(b) a}\right| U\right) \leqslant \frac{3}{5}\left(v_{b}^{\prime}-v_{b}\right)+v_{b} .
$$

By property (B2) (Proposition 4), there is an $a^{\prime} \geqslant a$ such that for any $a^{\prime \prime} \geqslant a^{\prime}$ one has

$$
p_{a a^{\prime \prime}}\left(X_{a^{\prime \prime}}\right) \subseteq U
$$

and therefore,

$$
d\left(f_{b} p_{f(b) a} p_{u a^{\prime \prime}}, f_{b}^{\prime} p_{f^{\prime}(b) a} p_{a a^{\prime \prime}}\right) \leqslant \frac{3}{5}\left(v_{b}^{\prime}-v_{b}\right)+v_{b} .
$$

Now (4), (5) and (11) yield the desired relation

$$
d\left(f_{b} p_{J^{\prime}(b) a^{\prime \prime}}, f_{b}^{\prime} p_{f^{\prime}(b) a^{\prime \prime}}\right) \leqslant v_{b}^{\prime} .
$$

Remark 5. By Proposition 4, (1) implies (3).

The next two lemmas show that certain modification of approximate mappings, called shifts, produce contiguous approximate mappings.

LEMMA 5. Let $f: X \rightarrow Y=\left(Y_{b}, v_{b}, q_{b b^{\prime}}, B\right)$ be an approximate mapping and let $v_{b}^{\prime}>v_{b}, b \in B$, be admissible meshes. If $B$ is cofinitc, there exists a function $\varphi: B \rightarrow A$, $\varphi \geqslant f$, such that any function $f^{\prime}: B \rightarrow A, f^{\prime} \geqslant \varphi$, together with the maps

$$
f_{b}^{\prime}=f_{b} p_{f(b) f^{\prime}(b)}: X_{f^{\prime}(b)} \rightarrow Y_{b}, \quad b \in B,
$$

form an approximate mapping $f^{\prime}=\left(f^{\prime}, f_{b}^{\prime}\right): X \rightarrow Y^{\prime}=\left(Y_{b}, v_{b}^{\prime}, q_{b b^{\prime}}, B\right)$, called an initial shift of $f$. The approximate mappings $f, f^{\prime}: X \rightarrow Y^{\prime}$ are contiguous.

Proof. For each $b \in B$ choose a number $\eta_{b}>0$ such that

$$
d\left(x, x^{\prime}\right) \leqslant \eta_{b} \Rightarrow d\left(q_{b_{0} b} f_{b}(x), q_{b_{b} b} f_{b}\left(x^{\prime}\right)\right) \leqslant \frac{1}{2}\left(v_{b_{0}}^{\prime}-v_{b_{0}}\right),
$$

for all $b_{0} \leqslant b$.

By (A2), cach $b \in B$ admits an index $\varphi(b) \geqslant f(b)$ such that for any $a^{\prime} \geqslant a \geqslant \varphi(b)$,

$$
d\left(p_{f(b) a} p_{a a^{\prime}}, p_{f(b) a^{\prime}}\right) \leqslant \eta_{b} .
$$

Let $f^{\prime}: B \rightarrow A$ satisfy $f^{\prime} \geqslant \varphi$. Put

$$
f_{b}^{\prime}=f_{b} p_{f(b) f^{\prime}(b)}, \quad b \in B
$$


If $b_{1} \leqslant b_{2}$, there is an $a \geqslant f\left(b_{1}\right), f\left(b_{2}\right)$ such that for any $a^{\prime} \geqslant a$ one has

$$
d\left(f_{b_{1}} p_{S\left(b_{1}\right) a^{\prime}}, q_{b_{1} b_{2}} f_{b_{2}} p_{S\left(b_{2}\right) a^{\prime}}\right) \leqslant v_{b_{1}} .
$$

We can choose $a \geqslant f^{\prime}\left(b_{1}\right), f^{\prime}\left(b_{2}\right)$. Then, by (15) and (14) for any $a^{\prime} \geqslant a$ one has

$$
\begin{gathered}
d\left(f_{b_{1}} p_{f\left(b_{1}\right) f^{\prime}\left(b_{1}\right)} p_{f^{\prime}\left(b_{1}\right) a^{\prime}}, f_{b_{1}} p_{f\left(b_{1}\right) a^{\prime}}\right) \leqslant \frac{1}{2}\left(v_{b_{1}}^{\prime}-v_{b_{1}}\right), \\
d\left(q_{b_{1} b_{2}} f_{b_{2}} p_{f\left(b_{2}\right) f^{\prime}\left(b_{2}\right)} p_{f^{\prime}\left(b_{2}\right) a^{\prime}}, q_{b_{1} l_{2}} f_{b_{1} p_{2}} p_{f\left(b_{2}\right) a^{\prime}}\right) \leqslant \frac{1}{2}\left(v_{b_{1}}^{\prime}-v_{b_{1}}\right) .
\end{gathered}
$$

(16), (18), (17) and (19) imply

$$
d\left(f_{b_{1}}^{\prime} p_{f^{\prime}\left(b_{1}\right) a^{\prime}}, q_{b_{1} b_{2}} f_{b_{2}}^{\prime} p_{\left.f^{\prime}\left(b_{3}\right) a_{a^{\prime}}\right)}\right) \leqslant v_{b_{1}}^{\prime},
$$

which shows that $f^{\prime}: X \rightarrow Y^{\prime}$ is indeed a mapping of approximate systems. Obviously, $f \equiv f^{\prime}$.

LBMma 6. Let $f: X \rightarrow Y=\left(Y_{b}, v_{b}, q_{b b}, B\right)$ be an approximate mapping and let $v_{b}^{\prime}>v_{b}, b \in B$, be admissible meshes. If $B$ is cofinite, there exists a function $\chi: B \rightarrow B$, $\chi(b) \geqslant b, b \in B$, such that for any function $\psi: B \rightarrow B, \psi \geqslant \chi$, the function $f^{\prime}=f \psi$ and the maps

$$
f_{b}^{\prime}=q_{b \psi(b)} f_{\psi(b)}: X_{f^{\prime}(b)} \rightarrow Y_{b}, \quad b \in B
$$

form an approximate mapping $f^{\prime}=\left(f^{\prime} f_{b}^{\prime}\right): X \rightarrow Y^{\prime}=\left(Y_{b}, v_{b}^{\prime}, q_{b b^{\prime}}, B\right)$, called a terminal shift of $f$. The approximate mappings $f^{\prime}, f^{\prime}: X \rightarrow Y^{\prime}$ are continuous.

Proof. Since $B$ is cofinite, by (A2), each $b \in B$ admits an index $\chi(b) \geqslant b$ such that, for $b^{\prime \prime} \geqslant b^{\prime} \geqslant \chi(b)$,

$$
d\left(q_{b b^{\prime}} q_{b^{\prime} b^{\prime \prime}}, q_{b b^{\prime \prime}}\right) \leqslant \frac{1}{4}\left(v_{b}^{\prime}-v_{b}\right)
$$

and even

$$
d\left(q_{b_{0} b} q_{b b^{\prime}} q_{b^{\prime} b^{\prime \prime}}, q_{b_{0} b} q_{b b^{\prime}}\right) \leqslant \frac{1}{4}\left(v_{b_{0}}^{\prime}-v_{b_{0}}\right)
$$

for all $b_{0} \leqslant b$.

By (A3), one can also assume that for $b^{\prime \prime} \geqslant \chi(b)$

$$
d\left(y, y^{\prime}\right) \leqslant v_{b^{\prime \prime}} \Rightarrow d\left(q_{b_{0} b} q_{b b^{\prime \prime}}(y), q_{b_{0} b} q_{b b^{\prime \prime}}\left(y^{\prime}\right)\right) \leqslant \frac{1}{4}\left(v_{b_{0}}^{\prime}-v_{b_{0}}\right)
$$

for all $b_{0} \leqslant b$.

We will now show that $\chi$ has the desired property. Let $\psi: B \rightarrow B$ be a function with $\psi \geqslant \chi$. Let $b_{1} \leqslant b_{2}$. Choose $b \geqslant \psi\left(b_{1}\right), \psi\left(b_{2}\right)$. By (AM1), there exists an index $a \geqslant f \psi\left(b_{1}\right), f \psi\left(b_{2}\right), f(b)$ such that for any $a^{\prime} \geqslant a$ one has

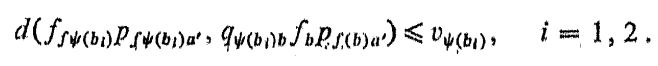

Since $\psi\left(b_{i}\right) \geqslant \chi\left(b_{i}\right),(25)$ and $(24)$ imply

(26) $\quad d\left(q_{b_{1} \psi\left(b_{1}\right)} f_{\psi\left(b_{1}\right)} p_{S \psi\left(b_{1}\right) a^{\prime}}, q_{b_{1} \psi\left(b_{1}\right)} q_{\psi\left(b_{1}\right) b} f_{b} p_{f(b) a^{\prime}}\right) \leqslant \frac{1}{4}\left(v_{b_{1}}^{\prime}-v_{b_{1}}\right)$,

(27) $\quad d\left(q_{b_{1} b_{2}} q_{b_{2} \psi\left(b_{2}\right)} f_{\psi\left(b_{2}\right)} p_{S \psi\left(b_{2}\right) a^{\prime}}, q_{b_{1} b_{2}} q_{b_{2} \psi\left(b_{2}\right)} q_{\psi\left(b_{2}\right) b} f_{b} p_{S(b) a^{\prime}}\right) \leqslant \frac{1}{4}\left(v_{b_{1}}^{\prime}-v_{b_{1}}\right)$.
By (22) and (23) we also have

$$
d\left(q_{b_{1}\left(\psi\left(b_{1}\right)\right.} q_{\psi\left(b_{1}\right) b} f_{b} p_{f(b) a^{\prime}}, q_{b_{1} b} f_{b} p_{f(b) a^{\prime}}\right) \leqslant \frac{1}{4}\left(v_{b_{1}}^{\prime}-v_{b_{1}}\right) ;
$$$$
d\left(q_{b_{1} b_{2}} q_{b_{2} \psi /\left(b_{2}\right)} q_{\psi\left(b_{2}\right) b} f_{b} p_{S(b) a^{\prime}}, q_{b_{1} b_{2}} q_{b_{2} b} f_{b} p_{S(b) a^{\circ}}\right) \leqslant \frac{1}{4}\left(v_{b_{1}}^{\prime}-v_{b_{1}}\right)
$$

Also, by (A1), we have

$$
d\left(q_{b_{1} b_{2}} q_{b_{2} b} f_{b} p_{f(b) d^{\prime}}, q_{b_{1} b} f_{b} p_{f(b) a^{\prime}}\right) \leqslant v_{b_{1}}
$$

Now (21), (26), (28), (30), (29) and (27) yield

$$
d\left(f_{b_{1}}^{\prime} p_{f^{\prime}\left(b_{1}\right) a^{\prime}}, q_{b_{1} b_{2}} f_{b_{2}}^{\prime} p_{S^{\prime}\left(b_{2}\right) a^{\prime}}\right) \leqslant v_{b_{1}}^{\prime},
$$

which shows that $\boldsymbol{f}^{\prime}=\left(f^{\prime}, f_{b}^{\prime}\right)$ is indeed an approximate mapping $\boldsymbol{f}^{\prime}: \boldsymbol{X} \rightarrow \boldsymbol{Y}^{\prime}$.

By (AM1) applied to $b \leqslant \psi(b)$, we see that there is an $a \geqslant f(b), f^{\prime}(b)=f \psi(b)$ : such that for $a^{\prime} \geqslant a$ one has

$$
d\left(f_{b}^{\prime} p_{f(b) a^{\prime}}, f_{b}^{\prime} p_{f^{\prime}(b) a^{\prime}}\right) \leqslant \dot{v}_{b} \leqslant \dot{v}_{b}^{\prime} .
$$

In $\S 6$ we will need the following lemma.

LeMma 7. Let $\boldsymbol{f}=\left(f, f_{b}\right): X \rightarrow \boldsymbol{X}=\left(Y_{b}, v_{b}, q_{b b^{\prime}}, B\right)$ be an approximate mapping and let $v_{b}^{\prime}>v_{b}, b \in B$, be admissible meshes. If $B$ is cofinite and $\delta_{b}>0$ are arbitrary numbers, there exists an approximate mapping $f^{\prime}=\left(f^{\prime}, f_{b}^{\prime}\right): X \rightarrow \boldsymbol{Y}^{\prime}$ $=\left(Y_{b}, v_{b}^{\prime}, q_{b b^{\prime}}, B\right)$ such that

$$
d\left(x, x^{\prime}\right) \leqslant u_{f^{\prime}(b)} \Rightarrow d\left(q_{h_{0} b} f_{b}^{\prime}(x), q_{b_{0} b} f_{b}^{\prime}\left(x^{\prime}\right)\right) \leqslant \delta_{b_{0}},
$$

for all $b_{0} \leqslant b$. Moreover, $f^{\prime} \equiv f: X \rightarrow Y^{\prime}$ and $f^{\prime \prime}$ is an initial shift of $\boldsymbol{f}$.

Proof. By uniform continuity and cofiniteness of $B$, for each $b \in B$, there is : a number $\eta_{b}>0$ such that

$$
\text { (34) } \quad d\left(x, x^{\prime}\right) \leqslant \eta_{b} \Rightarrow d\left(q_{b_{0} b} f_{b}(x), q_{b_{0} b} f_{b}\left(x^{\prime}\right)\right) \leqslant \delta_{b_{0}}, \quad b_{0} \geqslant b .
$$

By (A3), every $b \in B$ admits an index $\alpha(b) \geqslant f(b)$ such that, for any $a \geqslant \alpha(b)$,

$$
d\left(x, x^{\prime}\right) \leqslant u_{a} \Rightarrow d\left(p_{f^{\prime}(b) a}(x), p_{f(b) a}\left(x^{\prime}\right)\right) \leqslant \eta_{b} .
$$

We now choose a function $f^{\prime}: B \rightarrow A$ such that $f^{\prime} \geqslant \alpha$ and $f^{\prime} \geqslant \varphi$, where $\varphi$ is as in Lemma 5. Then $f^{\prime}$ and the maps $f_{b}^{\prime}=f_{b} p_{S(b) f^{\prime}(b)}$ form an approximate mapping $f^{\prime}: X \rightarrow Y^{\prime}$ and $f^{\prime}=f$. Moreover, (33) holds because of (34) and (35).

The relation of contiguity generates an equivalence relation in the set of all approximate mappings between two approximate systems.

Definirion 7. Let $\boldsymbol{X}$ and $\boldsymbol{Y}$ be two approximate systems. Approximate maps $f, f^{\prime}: X \rightarrow Y$ are called equivalent, written $f \sim f^{\prime}$, provided there is a finite collection of approximate maps $f_{i}: X \rightarrow Y, i=0 ; 1, \ldots ; n$; such that $f_{0}=f, f_{n}=f^{\prime}$ and $f_{i} \equiv f_{i+1}, i=0, \ldots, n-1$. 
An analogous definition applies to almost commutative maps between almost commutative systems.

The equivalence class containing $f$ will be denoted by $[f]$.

The main result of this section is the following theorem.

THEorem 4. Let $f, f^{\prime}: X \rightarrow Y$ be almost commutative mappings between almost commutative systems with limits $f, f^{\prime}: X \rightarrow Y$ respectively. If $B$ is cofinite, then $f=f^{\prime}$ if and only if $\boldsymbol{f} \sim \boldsymbol{f}^{\prime}$.

The sufficiency immediately follows from Definition 6 and Theorem 2. This part of the proof does not require the cofiniteness of $B$.

The necessity follows from the next lemma.

LEMMA 8. Let $f, f^{\prime}: X \rightarrow Y=\left(Y_{b}, v_{b}, q_{b b^{\prime}}, B\right)$ be approximate mappings with limits $f, f^{\prime}: X \rightarrow Y$ respectively. If $B$ is cofinite and $v_{b}^{\prime}>v_{b}, b \in B$, are admissible meshes, then $f=f^{\prime}$ implies $f \sim f^{\prime}: X \rightarrow Y^{\prime}=\left(Y_{b}, v_{b}^{\prime}, q_{b b^{\prime}}, B\right)$.

Proof. Choose functions $\chi, \chi^{\prime}: B \rightarrow B$ by applying Lemma 6 to $f$ and $f^{\prime}$ respectively. By (A3), there exists a function $\psi: B \rightarrow B$ such that $\psi \geqslant \chi, \chi^{\prime}$ and

$$
d\left(y, y^{\prime}\right) \leqslant v_{\psi(b)} \Rightarrow d\left(q_{b \psi(b)}(y), q_{b \psi(b)}\left(y^{\prime}\right)\right) \leqslant \frac{1}{2} v_{b}, \quad b \in B .
$$

Put $g=f \psi, g^{\prime}=f^{\prime} \psi$ and

$$
\begin{aligned}
& g_{b}=q_{b \psi(b)} f_{\psi(b)}, \\
& g_{b}^{\prime}=q_{b \psi(b)} f_{\psi(b)}^{\prime} .
\end{aligned}
$$

By Lemma $6, \boldsymbol{g}=\left(g, g_{b}\right), \boldsymbol{g}^{\prime}=\left(g^{\prime}, g_{b}^{\prime}\right)$ are approximate mappings $\boldsymbol{g}, \boldsymbol{g}^{\prime}: \boldsymbol{X} \rightarrow \boldsymbol{Y}^{\prime}$ and $\boldsymbol{f} \equiv \boldsymbol{g}, \boldsymbol{f}^{\prime} \equiv \boldsymbol{g}^{\prime}$. Moreover, by (LM),

$$
d\left(f_{\psi(b)} p_{S \psi(b)}, q_{\psi(b)} f\right) \leqslant v_{\psi(b)},
$$

and therefore, by (35) and (36),

$$
d\left(g_{b} p_{g(b)}, q_{b \psi(b)} q_{\psi(b)} f\right) \leqslant \frac{1}{2} v_{b}, \quad b \in B
$$

Analogously, (35) and (37) imply

$$
d\left(g_{b}^{\prime} p_{\theta^{\prime}(b)}, q_{b \psi(b)} q_{\psi(b)} f^{\prime}\right) \leqslant \frac{1}{2} v_{b}, \quad b \in B .
$$

Therefore, one has

$$
d\left(g_{b} p_{g(b)}, g_{b}^{\prime} p_{g^{\prime}(b)}\right) \leqslant v_{b} \leqslant v_{b}^{\prime}, \quad b \in B .
$$

By Lemma $4, g$ and $g^{\prime}$ are contiguous approximate mappings $\boldsymbol{Y} \rightarrow \boldsymbol{Y}^{\prime}$. Since $\boldsymbol{f} \equiv \boldsymbol{g} \equiv \boldsymbol{g}^{\prime} \equiv \boldsymbol{f}^{\prime}$, we obtain the desired relation $\boldsymbol{f} \sim \boldsymbol{f}^{\prime}$.

\section{Composition of approximate mappings}

Definirion 8. Let $X, Y$ and $Z=\left(Z_{c}, w_{c}, r_{c c^{\prime}}, C\right)$ be approximate systems and let $\boldsymbol{f}=\left(f, f_{b}\right): X \rightarrow \boldsymbol{Y}, \boldsymbol{g}=\left(g, g_{c}\right): \boldsymbol{Y} \rightarrow \boldsymbol{Z}$ be approximate maps. Let $h=f g: C \rightarrow A$ and let

$$
h_{c}=g_{c} f_{\theta(c)}: X_{h(c)} \rightarrow Z_{c}, \quad c \in C .
$$

If $\boldsymbol{h}=\left(h, h_{c}\right): \boldsymbol{X} \rightarrow \boldsymbol{Z}^{\prime}=\left(Z_{c}, w_{c}^{\prime}, r_{c c^{\prime}}, C\right)$ is an approximate mapping for some admissible mesh $w_{c}^{\prime} \geqslant w_{c}$ and if $\lim \boldsymbol{h}=g f$, then $\boldsymbol{h}$ is called the composition of $f$ and $g$ (for the meshes $w_{c}^{\prime}$ ) and is denoted by $\boldsymbol{h}=\boldsymbol{g} \boldsymbol{f}$.

Remark 6. In general, $\boldsymbol{h}$ is not an approximate mapping $\boldsymbol{X} \rightarrow \boldsymbol{Y}$. Even if $w_{c}^{\prime}>w_{c}$, $\boldsymbol{h}$ may not be an approximate mapping $\boldsymbol{X} \rightarrow \boldsymbol{Z}^{\prime}$. Nevertheless, we will see that, for cofinite $C$, the composition $g f$ is often defined. This will enable us to define the composition of equivalence classes $[g][f]$, whenever $C$ is cofinite.

LemMA 9. Let $\boldsymbol{f}=\left(f, f_{b}\right): X \rightarrow \boldsymbol{Y}$ and $\boldsymbol{g}=\left(g, g_{c}\right): \boldsymbol{Y} \rightarrow \boldsymbol{Z}=\left(Z_{c}, w_{c}, r_{c c^{\prime}}, C\right)$ be approximate maps and let $w_{c}^{\prime}>w_{c}$ be arbitrary admissible meshes. If $C$ is cofinite, there exists an approximate mapping $g^{\prime}=\left(g^{\prime}, g_{c}^{\prime}\right): Y \rightarrow Z^{\prime}=\left(Z_{c}, w_{c}^{\prime}, r_{c c^{\prime}}, C\right)$ (initial shift of $g$ ) such that $\boldsymbol{g} \equiv \boldsymbol{g}^{\prime}$ and the composition $\boldsymbol{g}^{\prime} f: X \rightarrow \boldsymbol{Z}^{\prime}$ is a well-defined approximate mapping with

$$
\lim \left(g^{\prime} f\right)=g f,
$$

where $g=\lim g=\lim g^{\prime}$ and $f=\lim f$.

Proof. Choose numbers $w_{c}^{\prime \prime}, c \in C$, such that $w_{c}<w_{c}^{\prime \prime}<w_{c}^{\prime}$. Clearly, $Z^{\prime \prime}=\left(Z_{c}, w_{c}^{\prime \prime}, r_{c c^{\prime}}, C\right)$ is an approximate system. By Lemma 7 , applied to $g: Y \rightarrow Z, w_{c}^{\prime \prime}>w_{c}$ and $\delta_{c}=\frac{1}{2}\left(w_{c}^{\prime}-w_{c}^{\prime \prime}\right)$, there exists an approximate mapping $\boldsymbol{g}^{\prime}=\left(g^{\prime}, g_{c}^{\prime}\right): \boldsymbol{Y} \rightarrow \boldsymbol{Z}^{\prime \prime}$ (initial shift of $\boldsymbol{g}$ ) such that

$$
\text { (3) } d\left(y, y^{\prime}\right) \leqslant v_{g^{\prime}(c)} \Rightarrow d\left(r_{c_{0} c} g_{c}^{\prime}(y), r_{c_{0} c} g_{c}^{\prime}\left(y^{\prime}\right)\right) \leqslant \frac{1}{2}\left(w_{c_{0}}^{\prime}-w_{c_{0}}^{\prime \prime}\right) \text {, }
$$

for all $c_{0} \leqslant c$.

We will first show that $h^{\prime}=f g^{\prime}$ and the maps

$$
h_{c}^{\prime}=g_{c}^{\prime} f_{g^{\prime}(c)}, \quad c \in C,
$$

form an approximate mapping $h^{\prime}: X \rightarrow Z^{\prime}$. Indeed, if $c_{1} \leqslant c_{2}$, then there is a $b \geqslant g^{\prime}\left(c_{1}\right), g^{\prime}\left(c_{2}\right)$ such that

$$
d\left(g_{c_{1}}^{\prime} q_{g^{\prime}\left(c_{1}\right) b}, r_{c_{1} c_{2}} g_{c_{2}}^{\prime} q_{g^{\prime}\left(c_{2}\right) b}\right) \leqslant w_{c_{1}}^{\prime \prime} .
$$

Moreover, there is an $a \geqslant f g\left(c_{1}\right), f g\left(c_{2}\right), f(b)$ such that for any $a^{\prime} \geqslant a$ one has

$$
d\left(f_{g^{\prime}\left(c_{i}\right)} p_{f \theta^{\prime}\left(c_{i}\right) a^{\prime}} q_{g^{\prime}\left(c_{i}\right) b} f_{b} p_{f(b) a^{\prime}}\right) \leqslant v_{g^{\prime}\left(c_{i}\right)}, \quad i=1,2 .
$$

Note that (6) and (3) imply

(7) $\quad d\left(g_{c_{1}}^{\prime} f_{g^{\prime}\left(c_{1}\right)} p_{f g^{\prime}\left(c_{1}\right) a^{\prime}}, g_{c_{1}}^{\prime} q_{g^{\prime}\left(c_{1}\right) b} f_{b} p_{f(b) a^{\prime}}\right) \leqslant \frac{1}{2}\left(w_{c_{1}}^{\prime}-w_{c_{1}}^{\prime \prime}\right)$,

(8) $\quad d\left(r_{c_{1} c_{2}} g_{c_{2}}^{\prime} f_{g^{\prime}\left(c_{2}\right)} p_{f g^{\prime}\left(c_{2}\right) a^{\prime}}, r_{c_{1} c_{2}} g_{c_{2}}^{\prime} q_{g^{\prime}\left(c_{2}\right) b} f_{b} p_{f(b) a^{\prime}}\right) \leqslant \frac{1}{2}\left(w_{c_{1}}^{\prime}-w_{c_{1}}^{\prime \prime}\right)$. 
Now (4), (7), (5) and (8) yield

$$
d\left(h_{c_{1}}^{\prime} p_{h^{\prime}\left(c_{1}\right) a^{\prime}}, r_{c_{1} c_{2}} h_{c_{2}}^{\prime} p_{h^{\prime \prime}\left(c_{2}\right) a^{\prime}}\right) \leqslant w_{c_{1}}^{\prime},
$$

which shows that $\boldsymbol{h}^{\prime}: \boldsymbol{X} \rightarrow \boldsymbol{Y}^{\prime}$ is an approximate mapping.

If $f=\lim f$, by $(\mathrm{LM})$,

$$
d\left(f_{g^{\prime}(c)} p_{h^{\prime}(c)}, q_{g^{\prime}(c)} f\right) \leqslant v_{g^{\prime}(c)} .
$$

Therefore, by (3) and (4),

$$
d\left(h_{c}^{\prime} p_{h^{\prime}(\sigma)}, g_{c}^{\prime} q_{\theta^{\prime}(c)} f\right) \leqslant \frac{1}{2}\left(w_{c}^{\prime}-w_{c}^{\prime \prime}\right) .
$$

If $g=\lim g$, then $g^{\prime} \equiv g$ implies $g=\lim g^{\prime}$ (Theorem 3). "Therefore, by (LM),'

$$
d\left(g_{c}^{\prime} g_{g^{\prime}(c)}, r_{c} g\right) \leqslant w_{c}^{\prime \prime}
$$

Now, (11) and (12) yield

$$
d\left(h_{c}^{\prime} p_{h^{\prime}(c)}, r_{c} g f\right) \leqslant \frac{1}{2}\left(w_{c}^{\prime}+w_{c}^{\prime \prime}\right) \leqslant w_{c}^{\prime \prime},
$$

which shows that indeed

$$
\lim \boldsymbol{h}^{\prime}=g f^{\prime}
$$

Definition 9. Let $X, Y$ and $Z=\left(Z_{c}, w_{c}, r_{c c^{\prime}}, C\right)$ be approximate systems, where $C$ is cofinite. Let $w_{c}^{\prime}>w_{c}$ be arbitrary admissible meshes and let $Z^{\prime}=\left(Z_{c}, w_{c}^{\prime}, r_{c c^{\prime}}, C\right)$. The composition $[g][f]$ of equivalence classes of approximate mappings $f: X \rightarrow Y, g: Y \rightarrow Z$ is the equivalence class $\left[g^{\prime} f\right]$ of the approximate mapping $g^{\prime} f: X \rightarrow Z^{\prime}$, where $g^{\prime} \sim \boldsymbol{g}$ is chosen so that $\boldsymbol{g}^{\prime} \boldsymbol{f}$ is defined.

Remark 7. By Lemma 9 such $\boldsymbol{g}^{\prime}$ exists and $\lim \left(\boldsymbol{g}^{\prime} f\right)=g f$, because $\lim \boldsymbol{g}^{\prime}=\lim \boldsymbol{g}$. If $g^{\prime \prime} \sim g$ is another representative of the class $g$ such that $g^{\prime \prime} f$ is defined, then by Definition $8, \lim \boldsymbol{g}^{\prime \prime} \boldsymbol{f}=g f$. We now conclude, by Theorem 4 , that $\boldsymbol{g}^{\prime \prime} \boldsymbol{f} \sim \boldsymbol{g} \boldsymbol{f}$ and therefore, $\left[g^{\prime \prime} f\right]=\left[g^{\prime} f\right]$. This shows that $[g][f]$ is well defined and $\lim ([g][f])=g f$

For any approximate system $Y=\left(Y_{b}, v_{b}, q_{b b^{\prime}}, B\right)$ we define the identity mapping $\mathbf{1}_{\mathbf{Y}}: \boldsymbol{Y} \rightarrow \boldsymbol{Y}$. It is given by the identity $1_{B}: B \rightarrow B$ and by the identity maps $1_{b}: Y_{b} \rightarrow Y_{b}$. Condition (AM1) is an immediate consequence of (A1).

Trrorom 5. Almost commutative systems over cofinite index sets and equivalence classes of almost commutative maps between such systems form a category denoted by Ap-Inv.

Proof. The associativity law

$$
[h]([g][f])=([h][g])[f]
$$

follows from $h(g f)=(h g) f$ and Theorem 4. Sinilarly $\left[\mathbf{1}_{X}\right][g]=[g]$ and $[g]\left[\mathbf{1}_{X}\right]=[g]$ follows from $\mathbf{1}_{Y} g=g$ and $g 1_{X}=g$ respectively.

It is a consequence of Definition 8 that $\lim$ is a functor from Ap-Inv to the category $\mathscr{C}$ of compact Hausdorff spaces and continuous mappings. By Theorem 4 , $\operatorname{im}[\boldsymbol{f}]=\lim \left[\boldsymbol{f}^{\prime}\right]$ implies $[\boldsymbol{f}]=\left[\boldsymbol{f}^{\prime}\right]$, which shows that $\lim :($ Ap-Inv) $\rightarrow \mathscr{C}$ is a faithful functor.

Let (Ap-Inv) $)_{A N R}$ denote the full subcategory of Ap-Inv whose objects are almost commutative systems of compact ANR's over cofinite index sets. By Theorem 2, $\lim :(A p-\operatorname{Inv})_{\mathrm{ANR}} \rightarrow \mathscr{C}$ is a full functor.

Furthermore, every compact Hausdorff space $X$ is homeomorphic to the limit of an inverse system $\boldsymbol{X}$ of compact polyhedra over an unbounded cofinite index set (see e.g., [8], I, $\S 5.2$, Theorem 8 and I, $\$ 1.2$, Theorem 2). By Propositions 1 and $2, X$ can be viewed as an almost commutative system of compact ANR's. This shows that lim on Ap-Inv and (Ap-Inv) $)_{\text {ANR }}$ is a representable functor.

Summarizing, we obtain the following theorem.

THeOrEM 6. The functor $\mathrm{lim}$ is an equivalence of categories between (Ap-Inv) $\mathrm{ANR}$ and $\mathscr{C}$

7. Approximate systems and shape of compact spaces. The purpose of this section is to show that approximate systems of ANR's over cofinite sets can be used to study shape of compact Hausdorff spaces in the same way in which commutative ANRsystems were used in [7] (see also [8]).

In this section we are interested in approximate systems $X=\left(X_{a}, u_{a}, p_{a a^{\prime}}, A\right)$ of compact ANR's which satisfy the following homotopy conditions.

$$
\begin{aligned}
p_{a_{1} a_{2}} p_{a_{2} a_{3}} \simeq p_{a_{1} a_{3}}, & a_{1} \leqslant a_{2} \leqslant a_{3}, \\
p_{a_{1} a_{2}} p_{a_{2}} \simeq p_{a_{1}}, & a_{1} \leqslant a_{2} .
\end{aligned}
$$

The next lemma shows how to convert an arbitrary approximate system of ANR's into one having properties $(\mathrm{H})$ and $(\mathrm{LH})$

LEMMA 10. Let $\boldsymbol{X}=\left(X_{a}, v_{a}, p_{a a^{\prime}}, A\right)$ be an approximate system of compact ANR's. Then there exists an ordering $\leqslant^{\prime}$ on $A$ such that $a<^{\prime} a^{\prime}$ implies $a<a^{\prime}$, $A^{\prime}=\left(A, \leqslant^{\prime}\right)$ is directed and $\boldsymbol{X}^{\prime}=\left(X_{a}, v_{a}, p_{a a^{\prime}}, A^{\prime}\right)$ is an approximate system satisfying $(\mathrm{H})$ and $(\mathrm{LH})$. Moreover, $X^{\prime}=\lim X^{\prime}$ and $X=\lim X$ coincide and so do the natural projections $p_{a}^{\prime}: X^{\prime} \rightarrow X_{a}$ and $p_{a}: X \rightarrow X_{a}, a \in A$.

Proof. For every $a \in A$ there is an $\eta_{a}>0$ such that $\eta_{a}$-near maps into $X_{a}$ are homotopic ( $X_{a}$ is an ANR). By property (A2), there is a function $\varphi: A \rightarrow A, \varphi(a) \geqslant a$, such that

$$
d\left(p_{a_{1} a_{2}} p_{a_{2} a_{3}}, p_{a_{1} a_{3}}\right) \leqslant \eta_{a_{1}}
$$

whenever $a_{3} \geqslant a_{2} \geqslant \varphi\left(a_{1}\right)$.

We put $a_{1}<^{\prime} a_{2}$ provided $a_{2}>\varphi\left(a_{1}\right)$ and we put $a_{1} \leqslant{ }^{\prime} a_{2}$ provided $a_{1}<{ }^{\prime} a_{2}$ or $a_{1}=a_{2}$. Clearly, $a_{1}<^{\prime} a_{2}$ and $a_{2} \leqslant a_{3}$ imply $a_{1}<^{\prime} a_{3}$. Moreover, since $A$ is unbounded, every $a \in A$ admits an $a^{\prime} \in A$ such that $a<^{\prime} a^{\prime}$. Then, by Proposition 5 , $X^{\prime}=\left(X_{a}, v_{a}, p_{a a^{\prime}}, A^{\prime}\right)$ is an approximate system and $X^{\prime}=X, p_{a}^{\prime}=p_{a}, a \in A$. 
Note that $a_{1}<^{\prime} a_{2} \leqslant^{\prime} a_{3}$ implies $a_{1} \leqslant \varphi\left(a_{1}\right)<a_{2} \leqslant a_{3}$ so that (1) yields (H). Moreover, (1) and Proposition 4 imply

(2)

$$
d\left(p_{a_{1} a_{2}} p_{a_{2}}, p_{a_{1}}\right) \leqslant \eta_{a_{1}}, \quad a_{1}<^{\prime} a_{2},
$$

which yields $(\mathrm{LH})$.

Let $X=\left(X_{a}, p_{a a^{\prime}}, A\right)$ be an almost commutative system of compact ANR's with limit $X$ and projections $p_{a}$ and let $(\mathrm{H})$ and (LH) be satisfied. Then the homotopy functor converts $X$ to a system $H X=\left(X_{a},\left[p_{a a^{\prime}}\right], A\right)$ in the homotopy category. Moreover, the homotopy classes $\left[p_{a}\right]: X \rightarrow X_{a}, a \in A$, form a morphism $H_{p}: X \rightarrow H X$ of pro-HTop (for terminology and notation see [8]).

Approximate ANR-systems (over cofinite index sets) with properties $(\mathrm{H})$ and $(\mathrm{LH})$ can be used in shape theory just as commutative ANR-systems because of the following theorem, which generalizes [8], I, § 5.3, Theorem 9 .

THEOREM 7. Let $X=\left(X_{a}, p_{a a^{\prime}}, A\right)$ be an almost commutative system of compact ANR's, which has properties $(\mathrm{H})$ and $(\mathrm{LH})$. Let $X=\lim X$ and let $p_{a}: X \rightarrow X_{a}, a \in A$, be natural projections. Then $H p=\left(\left[p_{a}\right]\right): X \rightarrow H X=\left(X_{a},\left[p_{a a^{\prime}}\right], A\right)$ is an HTopexpansion of $X$.

Proof. We must verify the following two conditions (see [8], $\mathrm{x}, \S 4.1$ and $\S 5.3$, Lemma 3).

(E1) For every mapping $h: X \rightarrow P$ into a compact ANR $P$ there exist an $a \in A$ and a mapping $f: X_{a} \rightarrow P$ such that $h \simeq f p_{a}$.

(E2) If $P$ is a compact ANR, $a \in A$ and $f, f^{\prime}: X_{a} \rightarrow P$ are mappings such that $f p_{a} \simeq f^{\prime} p_{a}$, then there exists an $a^{\prime} \geqslant a$ such that $f p_{a a^{\prime}} \simeq f^{\prime} p_{a a^{\prime}}$.

Proof of (E1). Choose $\eta>0$ so small that $\eta$-near maps into $P$ are homotopic By Property (R1) (Proposition 7), there exists an $a \in A$ and a mapping $f: X_{a} \rightarrow P$ such that $d\left(f p_{a}, h\right) \leqslant \eta$. Consequently, $f p_{a} \simeq h$.

Proof of (E2). Let $H: X \times I \rightarrow P$ be a homotopy with $H_{0}=f p_{a}$ and $H_{1}=f^{\prime} p_{a}$. By compactness, there are numbers $0=t_{0}<t_{1}<\ldots<t_{k}=1$ such that

$$
d\left(H_{t_{1}}, H_{t_{1+1}}\right) \leqslant \eta / 4, \quad i=0, \ldots, k-1 .
$$

By property (R1) (Proposition 7), there is an $a \in A$ and there are maps $f_{i}: X_{a} \rightarrow P$ such that

$$
d\left(H_{t}, f_{i} p_{a}\right) \leqslant \eta / 4, \quad i=0, \ldots, k \cdots-1
$$

(3) and (4) imply

$$
d\left(f_{i} p_{a}, f_{i+1} p_{a}\right)<\eta, \quad i=0, \ldots, k-1 .
$$

By Property (R2) (Proposition 7), there is an $a^{\prime} \geqslant a$ such that

and therefore,

$$
d\left(f_{i} p_{a a^{\prime}}, f_{i+1} p_{a a^{\prime}}\right)<\eta, \quad i=0, \ldots, k-1 .
$$

$$
f p_{a a^{\prime}}=f_{0} p_{a a^{\prime}} \simeq f_{1} p_{a a^{\prime}} \simeq \ldots \simeq f_{k} p_{a a^{\prime}}=f^{\prime} p_{a a^{\prime}}
$$

\section{References}

[1] Q. Haxhibeqiri, Shape fibrations for compact Hausdorff spaces, Publ. Inst. Math. (Beograd) (N.S) 31 (45) (1982), 33-49.

[2] S. Mardešić, Mappings of inverse systems, Glasnik Mat.-Fiz. Astron. 18 (1963), 241-254.

[3] - Approximate polyhedra, resolutions of maps and shape fibrations, Fund. Math. 114 (1981), 53-78.

[4] S. Mardešić and L. R. Rubin, Approximate inverse systems of compacta and covering dimensions, Pacific J. Math. 138 (1989), 129-144.

[5] - Cell-like mappings and non-metrizable compacta of finite cohomological dimension, Trans. Amer. Math. Soc. 313 (1989), 53-79.

[6] S. Mardešić and T. B. Rushing, Shape fibrations I, General Topology Appl. 9 (1978), $193-215$.

[7] S. Mardešić and J. Segal, Shapes of compacta and ANR-systems, Fund. Math. 72 (1971), 41-59.

[8] - - Shape Theory, North-Holland Publ. Co., Amsterdam 1982.

[9] - - PP-like continua and approximate inverse limits, Math. Japonica 33 (1988), 895-908.

[10] - Stability of almost commutative inverse systems of compacta, Topology Appl. 31 (1989), 285-299.

[10] - - Stability of almost commutative inverse systems of compacta, Topology Appl., to appear

[11] J. Mioduszewski, Mappings of inverse limits, Colloquium Math. 10 (1963), 39-44.

[12] T. Watanabe, Approximate shape, I, Basic notions, Tsukuba J. Math. 11 (1987), 17-59.

[13] - Approximate expansions of maps into inverse systems, in Geometric and Algebraic Topology, Banach Center Publ. 18 (1986), 363-370.

UNIVERSTTY OF ZAGREB

UNIVERSTY OF ZAGREB

Yugoslavia

UNIVERSITY OF WASHINGTON

Seattle, Washington 98195

Received 29 February 1988 